\title{
IMAGEN CORPORAL Y PERCEPCIÓN DE LA INFLUENCIA DE LOS MEDIOS DE COMUNICACIÓN: DIFERENCIAS DE GÉNERO EN UNA MUESTRA DE ADOLESCENTES*
}

\author{
Viviane Oliveira Gonçalves \\ Universidade Federal de Goiás, Goiânia, Goiás, Brasil \\ Juan Parra Martínez \\ Universidad de Castilla-La Mancha, Toledo, Espanha
}

\begin{abstract}
Resumen: El propósito del estudio es analizar las diferencias de género en una muestra de adolescentes respecto a la percepción de la imagen corporal, la percepción de la influencia de los medios de comunicación y la práctica de actividad física. Fue realizada una investigación con enfoque cualitativo, en la ciudad de Toledo (España) y el grupo de discusión se erigió como la técnica para la obtención de datos. Fueron constatadas diferencias en los discursos sobre el cuerpo de acuerdo al género y la existencia de mayor preocupación e insatisfacción con la imagen corporal en las adolescentes, y una gran diversidad de mediadores en la construcción de la imagen corporal en la actualidad: la norma de la delgadez, la inexistencia de una diversidad de tallas que se adapten a los diferentes cuerpos y la evidencia social de que las personas delgadas son más aceptadas en todos los ámbitos sociales.

Palabras Claves: Imagen corporal. Género. Medios masivos de comunicación.
\end{abstract}

\section{INTRODUCCIÓN}

Schilder (1999) define la imagen corporal como la representación mental del cuerpo que cada individuo construye en su mente. Actualmente, se entiende como un constructo multidimensional, que incluye: la dimensión

\footnotetext{
* Artigo recebido em 1\%/12/2013 e aprovado em 5/5/2014.
} 
perceptiva (precisión en la percepción); la dimensión cognitiva-afectiva o subjetiva (actitudes, sentimientos, cogniciones y valoraciones en relación al tamaño corporal, peso o partes del cuerpo) y la dimensión conductual (conductas que la percepción del cuerpo y los sentimientos asociados provocan). Por tanto, para Cash y Pruzinsky (1990), en la experiencia dinámica de la imagen corporal en la vida cotidiana deben ser considerados el rol de situaciones y los contextos emocionales, así como las fluctuaciones individuales relacionadas con atributos psicológicos y características físicas del momento.

Tavares (2003) también afirma que la imagen corporal es construida a partir del contacto con la realidad externa. Ese contacto es una experiencia activa en que unas partes son aceptadas y otras rechazadas. En este sentido, afirma que la imagen del cuerpo nunca es estática o completa, ya que hay tendencias a la ruptura y a la reestructuración conforme los cambios experimentados a lo largo de la vida.

La importancia de la imagen corporal ha sido una constante a lo largo de la historia del ser humano, ha variado en diferentes culturas y ha tenido más o menos incidencia según el modelo social. En la actualidad cobra mayor repercusión debido a la gran influencia que ejercen los medios de comunicación y la publicidad, y la facilidad con la que se transmiten los modelos corporales a través de las nuevas tecnologías. Sin embargo, los modelos corporales, que se nos presentan como referencia, no son iguales para un sexo y otro. El componente de género ejerce una gran presión sociocultural que no afecta de la misma manera a la mujer que al hombre.

De acuerdo con San Miguel (2002), el género no hace referencia simplemente a las diferencias relativas al sexo. El término sexo nos remite a las diferencias biológicas, anatómicas, cromosómicas y fisiológicas que distinguen entre sí al hombre y a la mujer; mientras que género se refiere a la construcción cultural que se realiza sobre esas diferencias, es decir, el proceso de socialización por el que cada sujeto asume las pautas de comportamiento y las perspectivas propias de su sexo. Esta construcción sociocultural sobre una base biológica se conoce con el nombre de sistema sexo-género y se convierte en un elemento estructural de toda sociedad. Según Murillo (2000), el sistema sexo-género permite conocer un modelo de sociedad en el que se explica cómo las diferencias biológicas entre las mujeres y los hombres se han traducido históricamente en desigualdad de índole social, política y económica, siendo las mujeres las más desfavorecidas en este proceso.

Queremos resaltar, además, que a la categoría de género se articulan otras instancias que permiten entender la forma en que se organiza la 
dimensión de género en la subjetividad de los individuos. Estas instancias son los roles de género, los estereotipos de género y la identidad de género.

Los roles o papeles de género se refieren a las normas y expectativas socioculturales de comportamientos y actividades que son considerados como apropiados y deseables para hombres y mujeres (PARRA MARTíNEZ, 2002). Dentro de esta perspectiva se habla también de la división sexual del trabajo entre hombres y mujeres, es decir, las actividades y tareas tienden a estar divididas, en función de los roles que tradicionalmente se le han establecido, asignándoles a las mujeres las tareas de la casa y el cuidado de los niños, mientras se espera de los hombres que adopten más actividades de posición o estatus en la comunidad.

Los estereotipos son aquellas creencias, valores y normas que llevan implícitas una definición social que, al ser aceptadas por la mayoría de los individuos en la sociedad, reflejan los papeles que son asignados a hombres y mujeres (MINA-FREIRE, 2005). De esta manera, en la mayoría de las sociedades se espera que los hombres muestren muchos comportamientos caracterizados como agresivos, autónomos, y dominantes; de las mujeres se espera que muestren comportamientos de cuidado, complacencia y pasividad.

La identidad de género, a su vez, corresponde al sentimiento de pertenencia al sexo femenino o masculino. Según Sheriff (1982), la identidad de género hace referencia al hecho de percibir y vernos a nosotros mismos como mujer o como varón, dando como resultado el rol o papel de género que es la expresión de la masculinidad o feminidad teniendo en cuenta las normas establecidas por la sociedad. En este sentido, la identidad de género es el componente del autoconcepto que se adquiere más temprano, a partir del mensaje familiar y social junto a la propia imagen corporal, siendo el más central y el de mayor capacidad organizativa, debido a la relevancia que concede la sociedad a todo aquello relacionado con el sexo.

La construcción de la identidad en la contemporaneidad incorpora de forma demasiada la apariencia, la imagen y el consumo, material e inmaterial, que establece y determina las construcciones de la apariencia y de la imagen establecida como ideal. La existencia de este ideal de belleza, establecido y compartido socialmente, supone una presión significativa para la población en general y, especialmente, para el adolescente que se encuentra en la etapa de integración de la imagen corporal. Esta integración es un proceso que se desarrolla a lo largo del tiempo y tiene claras influencias sociales. Es decir, la aceptación del propio cuerpo va a estar condicionada por los criterios predominantes en el grupo de pares, quienes están, a su vez, determinados 
por los modelos sociales en la actualidad. Así, los y las adolescentes se encuentran presionados por múltiples influencias de la sociedad: publicidad, prensa, televisión, familiares, amistades etc., que les proponen un cuerpo delgado y esbelto, sobre todo en el ámbito femenino.

De hecho, al revisar la literatura en torno a la relación imagen corporal y los medios de comunicación masivos, se aprecia con claridad que la mayor parte de la investigación realizada es con mujeres adolescentes y adultas jóvenes, en quienes suele acentuarse la preocupación por la apariencia física (WIKES y GUNTER, 2005; CALADO et al., 2011). De igual forma, la insatisfacción corporal ha sido relacionada con baja autoestima, depresión y con el impulso inicial en el comportamiento de trastornos de la conducta alimentaria, especialmente en mujeres adolescentes y adultas jóvenes, ya que para esta población la apariencia está más centrada en el autoconcepto y la evaluación de los demás, y culturalmente la belleza ideal femenina es ser muy delgada, lo que para la mayoría es inalcanzable y poco saludable (BAILE AYENSA, GUILLÉN y GARRIDO, 2002). Cabe destacar, como sugieren Hargreaves y Tiggemann (2003), que se conoce poco sobre la exposición de los chicos a los ideales que promueven los medios de comunicación y la publicidad.

Por lo anterior, se ha planteado el presente estudio en torno a los siguientes interrogantes: ¿Cuáles son las diferencias de género respecto a la percepción de la imagen corporal? ¿Cuáles son los patrones más relevantes? ¿Qué valoración hacen de los patrones culturales estéticos divulgados por la cultura de consumo y de la imagen, y de los efectos que éstos tienen en la imagen corporal?

El propósito de este trabajo es aportar a la discusión de este tema, pues ahondar en el conocimiento sobre la influencia que los medios de comunicación tienen en la transmisión de estereotipos de género en torno al cuerpo y la belleza puede ser beneficiosa para entender la percepción y valoración de los cambios y las expectativas vinculadas a la imagen corporal durante el período de la pubertad y adolescencia.

\section{AsPeCtOS METODOLÓGICOS}

El objetivo general del estudio es investigar las diferencias de género en la percepción de la imagen corporal y la percepción de la influencia de los medios de comunicación en una muestra de adolescentes de la provincia de Toledo. Tiene un enfoque cualitativo, se basa en la investigación que produce datos descriptivos inferidos de las palabras habladas o escritas y de la conducta observable en el grupo de estudio (HERNÁNDEZ, FERNÁNDEZ y BAPTISTA, 2006). 
Dadas las características del estudio, el grupo de discusión se erigió como la técnica para la obtención de datos. Ibañez (1994) afirma que los grupos de discusión son una técnica de investigación que recolecta información derivada de la interacción de un grupo de personas con el objetivo de conocer sus pensamientos y sentimientos respecto al tema planteado por el investigador, quien con la aplicación de esta metodología logrará obtener resultados que no indican una respuesta simple y generalmente inexacta, sino un rango amplio de opiniones que orientan el análisis con mayor amplitud y alcance. Gutiérrez (2008) aclara que el término focus group es el más utilizado y conocido en el ámbito anglosajón, mientras que el término grupo de discusión tiene una referencia mayormente española. Los focus groups son focalizados en temas específicos y buscan respuestas a cuestiones concretas, en que la moderación es directiva y no necesariamente se estimula la interacción entre los participantes. Para Gutiérrez (2008) lo que diferencia el grupo de discusión de otras técnicas es el sentido reflexivo que no manifiestan otros instrumentos. Suponemos adecuada esta técnica por considerarse un procedimiento que es muy apropiado cuando uno de los objetivos del estudio es explicar cómo perciben los sujetos una experiencia, idea o hecho, como es el caso de la percepción de la imagen corporal.

Llevamos a cabo diez grupos de discusión en tres institutos de la provincia de Toledo: Toledo capital, Talavera de la Reina e Madridejos, totalizando una muestra de 60 participantes (de 14 a 18 años). En lo referente al número de participantes por grupo de discusión, este fue de seis personas (Tabla 1), lo que corresponde a lo sugerido por Alonso (1998), quien considera que el número adecuado se da a partir de cinco personas para evitar la intimidad que se genera en las conversaciones cuando son tres personas o la conversación por parejas cuando son cuatro. Ibáñez (1994) también señala que el número adecuado de participantes oscila entre cinco y diez personas.

Tabla 1. Perfil de los/las participantes de los grupos de discusión.

\begin{tabular}{|l|l|l|l|c|}
\hline & & Curso & Localidad & N \\
\hline Grupo 1 & sexo femenino & $3^{\circ} \mathrm{ESO}$ & Toledo & 06 \\
\hline Grupo 2 & sexo masculino & $3^{\circ} \mathrm{ESO}$ & Toledo & 06 \\
\hline Grupo 3 & sexo femenino & $4^{\circ} \mathrm{ESO}$ & Toledo & 06 \\
\hline Grupo 4 & sexo masculino & $4^{\circ} \mathrm{ESO}$ & Toledo & 06 \\
\hline Grupo 5 & sexo femenino & $3^{\circ} \mathrm{ESO}$ & Madridejos & 06 \\
\hline Grupo 6 & sexo masculino & $3^{\circ} \mathrm{ESO}$ & Madridejos & 06 \\
\hline Grupos 7 y 8 & sexo femenino & $1^{\circ} \mathrm{BACH}$ & Talavera & 12 \\
\hline Grupos 9 y 10 & sexo masculino & $1^{\circ} \mathrm{BACH}$ & Talavera & 12 \\
\hline
\end{tabular}


Antes de realizar los grupos, hicimos una visita a los centros para ponernos en contacto con los profesores/as de Educación Física y establecer la forma en que llevaríamos a la práctica los grupos. Los grupos de discusión se organizaron de forma voluntaria o a través de la indicación del profesorado de la asignatura Educación Física del centro educativo. Los diálogos de todos los grupos fueron registrados por medio de grabadoras de audio. Se optó por constituir un guion con preguntas abiertas, con una lista de temas que fue interiorizado y aprendido por la investigadora. Dicho guion de discusión inicial consideró los siguientes aspectos: 1) preámbulo para iniciar el grupo de discusión, y 2) subtítulos y preguntas del tema. Las preguntas se relacionan con: la percepción de su propio cuerpo, el ideal de belleza social, la presión social existente sobre la belleza física, la influencia de los medios de comunicación y la relación entre imagen corporal y la práctica de actividad física. Además, las preguntas fueron ordenadas de lo más general a lo más específico, es decir, comenzábamos con preguntas muy generales, que facilitaban la participación y rompían el hielo, y que poco a poco nos iban llevando a las cuestiones más específicas que queríamos estudiar.

Para llevar a cabo la formalización categorizada y codificada de los datos extraídos de las opiniones expresadas en el grupo de discusión, utilizamos un programa informático para el análisis de dados cualitativos - el ATLAS.ti. Si bien en nuestra investigación, partimos de una revisión bibliográfica preliminar y en la técnica de recolección de datos cualitativos contemplamos unas categorías o tópicos de análisis que conformaron el guion temático de los grupos de discusión, en el proceso de codificación optamos por la estrategia inductiva. De esta forma, no partimos de una lista de códigos previamente establecida, sino que los construimos en función de la información recogida.

Partiendo de la lectura de los discursos, se establecieron dos niveles progresivos de reducción y estructuración teórica de la información mediante: 1) segmentación e identificación de significado y agrupación en citas (unidades de contenido); y 2) codificación del texto, estableciendo códigos en vivo y agrupándolos según tópicos del tema. Se asignó un código de tres letras a cada cita y posteriormente a cada categoría, que coinciden, en general, con las primeras de la denominación que recibe la categoría o la cita. Realizado el proceso de codificación, se procedió a reagrupar los códigos por similitud de significado, lo cual dio lugar a la categorización. 
RESULTADOS Y DISCUSIÓN

\section{El listado de los códigos empleados y de las categorías y subcategorías resultantes lo mostramos a continuación:}

Tabla 2. Categorías, subcategorías y códigos emergentes.

\begin{tabular}{|c|c|c|}
\hline Categorías & Subcategorías & Códigos \\
\hline \multirow{4}{*}{ Imagen Corporal } & $\begin{array}{l}\text { Percepción del } \\
\text { cuerpo próprio }\end{array}$ & $\begin{array}{l}\text { - Sentimientos en relación al cuerpo (SIC) } \\
\text { - Satisfacción con áreas corporales (SAC) } \\
\text { - Insatisfacción con áreas corporales (IAC) } \\
\text { - Cambios físicos que gustaría hacer (CIC) }\end{array}$ \\
\hline & $\begin{array}{l}\text { Percepción del } \\
\text { cuerpo del otro }\end{array}$ & $\begin{array}{l}\text { - Percepción en relación al género femenino (PGF) } \\
\text { - Percepción en relación al género masculino (PGM) } \\
\text { - Interés o qué les llama la atención por el género femenino } \\
\text { de una forma general (IGF) } \\
\text { - Interés o qué les llama la atención por el género masculino } \\
\text { de una forma general (IGM) } \\
\text { - Interés/atención por el cuerpo masculino (ICM) } \\
\text { - Interés/atención por el cuerpo femenino (ICF) }\end{array}$ \\
\hline & $\begin{array}{l}\text { Preocupación por } \\
\text { la forma corporal }\end{array}$ & $\begin{array}{l}\text { - Preocupación por estar en forma (PFF) } \\
\text { - Preocupación por la forma física según el género (PFG) }\end{array}$ \\
\hline & $\begin{array}{l}\text { Cuidados del } \\
\text { cuerpo }\end{array}$ & $\begin{array}{l}\text { - Cosméticos y maquillaje (CRE) } \\
\text { - Diferencias en relación a los cuidados del cuerpo según el } \\
\text { género (DCG) } \\
\text { - Dieta como forma de mejorar la imagen corporal (CUD) } \\
\text { - Ejercicio físico como forma de mejorar la imagen corporal } \\
\text { (CUE) } \\
\text { - Cambios en relación a los cuidados del cuerpo por parte de } \\
\text { los hombres (CCH) } \\
\text { - Operaciones plásticas (OPP) } \\
\text { - Motivos para invertir en la imagen corporal (MOT) }\end{array}$ \\
\hline \multirow{3}{*}{$\begin{array}{l}\text { Percepción de } \\
\text { la influencia de } \\
\text { los Medios de } \\
\text { Comunicación }\end{array}$} & $\begin{array}{l}\text { Patrón corporal } \\
\text { en la sociedad de } \\
\text { consumo }\end{array}$ & $\begin{array}{l}\text { - Patrón corporal en distintas culturas y épocas (CDC) } \\
\text { - Norma de la delgadez (NDE) } \\
\text { - Las tallas (TLL) } \\
\text { - Belleza física como ventaja profesional (TVB) } \\
\text { - Percepción respecto a las personas que no se encuadran en } \\
\text { los patrones corporales de belleza (PPP) } \\
\text { - La cuestión de la auto-estima (ESI) }\end{array}$ \\
\hline & $\begin{array}{l}\text { Prototipos } \\
\text { corporales }\end{array}$ & $\begin{array}{l}\text { - Prototipos corporales masculinos (PCM) } \\
\text { - Prototipos corporales femeninos (PCF) } \\
\text { - Prototipos corporales transmitidos según el género (PCG) } \\
\text { - Modelos corporales (MOD) }\end{array}$ \\
\hline & $\begin{array}{l}\text { Influencia de } \\
\text { los medios de } \\
\text { comunicación }\end{array}$ & $\begin{array}{l}\text { - Percepción de la influencia de los medios de comunicación } \\
\text { (IMM) } \\
\text { - Mensajes trasmitidos por los medios de comunicación (MET) } \\
\text { - Mundo irreal presentado por los medios de comunicación } \\
\text { (MIT) } \\
\text { - Las mujeres como el principal objetivo de los medios de } \\
\text { - Comunicación (MAP) } \\
\text { - Percepción de la influencia de los medios de comunicación } \\
\text { - Según el género (IMG) } \\
\text { - Críticas a la influencia de los medios de comunicación en la } \\
\text { imagen corporal (CIM) }\end{array}$ \\
\hline
\end{tabular}




\section{Percepción de la imagen Corporal}

El primer aspecto que emerge en los discursos de los participantes con relación a la imagen corporal se refiere a la percepción del propio cuerpo. En esta dimensión confluyen diversos aspectos como sentimientos relacionados al cuerpo, satisfacción o insatisfacción con las áreas corporales, y cambios que les gustaría hacer. Respecto a los sentimientos en relación al propio cuerpo, mientras las chicas manifiestan sentimientos poco positivos, la mayoría de los chicos dicen sentirse bien, lo cual atribuyen a la práctica deportiva y a un estilo de vida más saludable.

En términos generales, las chicas participantes de los grupos de discusión afirman estar insatisfechas con el peso, con la parte inferior y la parte mediana del cuerpo. Los chicos manifestaron insatisfacción con el peso y el tono muscular.

Sí, a mí el pelo, el color de los ojos, estas cosas, me gusta más. Lo que no me gusta es la forma física. (1० Bach femenino)

Sí, yo creo que también hay chicas que a veces se fijan en las demás, en su forma de la cintura y de la tripa, si tiene un vientre plano, si tiene a lo mejor por aquí por la cadera. ( $1^{\circ}$ Bach femenino)

Yo no me hago mucho caso pero me gustaría ser más alta y más flaca. ( $3^{\circ}$ ESO femenino)

Bueno, cuanto al peso, un poco más de peso (...) Masa muscular más definida. (1० Bach masculino)

A mí me gustaría estar más definido o algo más definido, pero sin tomar nada. (4 ESO masculino)

Resaltamos que la insatisfacción corporal hallada en el grupo de chicos con relación al peso está más relacionada con el deseo de ganar peso y presentar una musculatura más definida, mientras que en las mujeres lo que se desea es perder peso, es decir, a las mujeres parece no existir espacio para la variedad de propuestas estéticas que no sea el ser joven y delgada. La delgadez es la única vía con la que van a establecer comparaciones, lo que puede elevar el nivel de insatisfacción corporal.

Según Tavares (2003), la imagen corporal se refiere a la representación mental del tamaño, figura y de la forma de nuestro cuerpo (en general y de sus partes), es decir, cómo lo vemos, pero también cómo creemos que los demás lo ven. De esa forma, en la subcategoría percepción del cuerpo del otro, presentamos la mirada de los participantes hacia sus pares y hacia el cuerpo del otro, lo que además nos ayuda a desvelar otros aspectos concernientes a las relaciones de género. 
La mayoría de los participantes reconocen que las diferencias entre géneros les son atribuidas social y culturalmente, es decir, que la sociedad sigue sometiendo a hombres y mujeres a estereotipos de género, describiendo sus calidades y conductas y decidiendo cómo deben ser y lo que deben hacer.

Lo que pasa es que nos exigen ser más disciplinadas y guapas. ( $3^{\circ} \mathrm{ESO}$ femenino)

A ver, es que a las mujeres les exigen que sean guapas y delgadas, mucho más que los chicos. ( $3^{\circ}$ ESO masculino)

Es que si te pones a pensar todo el tiempo te están diciendo lo que tienes que hacer y eso... en casa, en el Instituto, en las revistas, en todas partes. (3० ESO femenino)

Yo no creo que sea así, pero es verdad que hay eso de cuídate, y una chica no debe hacer esto y lo otro y no bebas y no fumes y ten cuidado. ( $3^{\circ} \mathrm{ESO}$ femenino)

Sí que es verdad y a los chicos no. (3० ESO femenino)

Sí, por la educación creo, la forma como te cría la familia. (4 ESO femenino) Sí, a las chicas les dan muñecas, y ollas y planchas y a los chicos coches, pelotas, juegos. (4 ESO masculino)

Cuando se pregunta, de forma general, sobre el interés por el sexo opuesto, las respuestas reflejan que chicos y chicas consideran la importancia del aspecto físico, aunque señalan también la importancia de los aspectos de la personalidad.

Pero primero te fijas en el estilo, luego te entra por los ojos, luego ya ves si de verdad te gusta o no. Pero siempre te vas a fijar primero en el físico. (4º ESO masculino)

Para mí sobre todo tiene que estar guapa para mí, ya que estén buenas o no, pero sobre todo que sea como sea, o sea, de carácter y eso. (4 ESO masculino) Una chica guapa, de buena figura, inteligente. Que no sea presumida, que no esté todo el rato "mira, que guapa soy", ni nada de eso. (1 Bach masculino)

Por otro lado, cuando se les plantea que manifiesten su interés solamente respecto al cuerpo del sexo opuesto, las respuestas reflejan de forma significativa las diferencias en el modelo corporal atribuido a cada sexo. El modelo corporal imperante sería el que cumpliría con las características apuntadas por Pérez Gauli (2002), es decir, mientras que en la representación del cuerpo masculino se priorizan los modelos con una complexión atlética, en el caso de las mujeres se destacan los cuerpos de una mayor delgadez. 
Yo creo que también, porque si va vestido enteramente, luego ya pues si tiene buenas piernas, si hace deporte, si se nota los músculos, lo típico, pero principalmente la cara. ( $1^{\circ}$ Bach femenino)

Yo también la cara, pero también que esté proporcionado, que si es muy alto, pero que no sea ahí un palo. (risas) (1º Bach femenino)

Pues morenita, ojos verdes, delgadita, altita... ( $3^{\circ}$ ESO masculino)

Gorda no. Porque a veces si es delgada es porque se cuida y tiene la cara bien y si no se cuida tiene la cara peor. ( $1^{\circ}$ Bach masculino)

Respecto a la preocupación con la forma corporal, la opinión de los participantes es de que las chicas suelen presentar una mayor preocupación con el cuerpo que los chicos.

Suelen preocuparse con lo que va a ver el otro, por lo que dirán. (1º Bach masculino)

Porque desde pequeñitas las están haciendo ver como que tienen que ser más guapas, están más preocupadas con eso, mientras que los hombres se conforman. (4 ESO masculino)

Yo creo que es una cosa mucho más de las chicas que de los chicos, porque a los chicos estar gordos no les importa. (3॰ ESO masculino)

Yo pienso lo mismo, los chicos yo creo que, a lo mejor que son pasotas, o que no se preocupan nada o no lo sé por qué. Lo más normal no, pero lo normal es que nos preocupemos, pero lo más sano y lo más decente, porque es que somos demasiado perfeccionistas en este tema y nos obsesionamos. ( $1 \circ$ Bach femenino)

En los discursos producidos por los grupos de discusión en la subcategoría cuidados con el cuerpo, observamos que la mayoría de los participantes practican actividad físico-deportiva y la dieta como forma de mejorar la apariencia, además de otros comportamientos como el uso de cosméticos y maquillaje. Por otra parte, afirman que la cirugía estética es más común entre las mujeres que entre los hombres.

Eso de operarse está más para las chicas. Las chicas son las que más se operan. Pero sí que te cambias mucho, pero cambias físicamente, porque si internamente eres así, no tienes nada que hacer. Pero, luego, físicamente sí que atraes más. ( $1^{\circ}$ Bach masculino)

Lo que se suelen operar son los pechos y luego se hacen liposucciones. (10 Bach masculino)

Observamos en la literatura que el tema ha sido abordado principalmente desde la sociología y la antropología. La perspectiva socioantropológica está representada por autores como Giddens (1997), Le Breton 
(2002) y Lipovetsky (1986). En estos autores se subraya la interpretación de la cirugía plástica como una práctica de la modernidad imbricada en las prácticas y discursos propios de la sociedad de consumo y del individualismo contemporáneo. Otro grupo que ha abordado el tema de la cirugía estética ha sido el feminista (Wolf, 1991). En este sentido, las mujeres que se someten a cirugía por razones puramente estéticas, han sido vistas como víctimas de la ideología patriarcal en la cual la autoestima de las mujeres depende de manera preponderante de su apariencia física. Además, el crecimiento en el uso de la cirugía plástica, preferentemente entre las mujeres, ha sido visto como un síntoma del permanente sentimiento de insatisfacción que la mayoría de las mujeres tiene con respecto a su apariencia física, en parte como consecuencia de la transmisión constante de imágenes de perfección por parte de los medios de comunicación.

\section{Percepción de la influencia de los medios de COMUNiCACIÓN}

A esta categoría están relacionados tres conceptos: patrón corporal en la sociedad de consumo, prototipos corporales y percepción de la influencia de los medios. El primero se refiere a la percepción de los y las participantes respecto a los patrones corporales en la sociedad actual y el segundo a los prototipos corporales transmitidos por los medios de comunicación de masas en función del género. Por fin, el tercer concepto comprende la percepción de la influencia de los medios de comunicación, así como el mundo presentado y los mensajes transmitidos a través de dichos medios.

En los medios de comunicación en general es posible observar un constante flujo de imágenes de hombres y mujeres regidos por unos cánones de belleza que son restrictivos a la mayoría de la población normal. Los participantes de los grupos de discusión identifican esos prototipos corporales transmitidos a través de los medios de comunicación, tal como observamos en algunas de sus respuestas, descritas a continuación.

Las chicas suelen ser anoréxicas o esqueléticas. (1 Bach masculino)

Delgadísimas las ponen siempre. $Y$ altísimas, con unas piernas muy altas. $Y$ si son bajitas, más delgadas aún, para que no se note. (1Bach masculino)

Cuando cuestionados respecto a los modelos corporales que tienen como referencia, los participantes de los grupos de discusión demuestran que la mayoría de las propuestas corporales consideradas ideales derivan principalmente de los medios de comunicación, aunque con algunas excepciones. Para unos, las mediaciones más destacadas para la construcción de la imagen corporal vienen dadas por la presencia de cantantes, actrices, 
por la publicidad y por la moda. Otros señalan la importancia de modelos corporales más cercanos y más reales, como parientes o amigos. Entre los nombres más citados están Sara Carbonero, Shakira, Fernando Torres, Angelina Jolie, los jugadores de fútbol, entre otros.

Opinan que su cultura es una cultura obsesionada por el cuerpo y su exposición visual, tecnológica y comercial. En consecuencia, hay una presión enorme para estar delgado/a y guapo/a, de manera que ser feo y principalmente estar gordo son obstáculos para el éxito social y profesional.

Pues yo estoy con ella, si eres guapa es más fácil porque las guapas les gustan a todos. ( $3^{\circ} \mathrm{ESO}$ femenino)

Yo también creo que influye, es difícil que tengas un trabajo si eres gorda, bueno, en la administración sí, porque no te pueden dejar y yo conozco porque mi madre trabaja en la administración, pero en la empresa, seguro que no te cogen. ( $3^{\circ}$ ESO masculino)

Con relación a los mensajes transmitidos, profundizando en el tipo de influencia que ejerce la televisión, los y las participantes explicitan que la belleza se valida en este medio. A ese respecto, aparecen interesantes apreciaciones que pueden ser resumidas en dos ideas: la belleza está asociada a la delgadez, y la belleza asociada al éxito social y a la felicidad.

Puede ser que... los anuncios son como que la vida perfecta, somos ideales de la muerte porque llevo este sujetador, porque soy muy guay, no sé. Si me compro el sujetador éste y en cima pues intento ser tan delgada y tan guapa y me pongo tantas cosas como se pone la modelo, pues a lo mejor soy más feliz. Como ella aparenta ser feliz, pues yo soy feliz. ( $1{ }^{\circ}$ Bach femenino)

Por otra parte, el análisis del discurso producido en los grupos de discusión refleja que la comparación del propio cuerpo con el cuerpo de los modelos de belleza corporal divulgados por los medios de comunicación suele estar relacionado a conductas de pérdida de peso $u$ otros cuidados del cuerpo.

Respecto a las diferencias de género en relación a los mensajes transmitidos por los medios de comunicación, chicos y chicas argumentan que son las mujeres el principal objetivo de los mensajes.

Pues por la cultura que tenemos, porque nos dicen los cánones que tienes que seguir, ponen los escaparates, los maniquíes tienen una 38 o una 36, con un pecho una 90 y tal. Hombre, pues es muy difícil no fijar en esto, porque se está rodeado completamente. Los chicos, no lo sé si es porque tiene menos maniquíes o menos publicidad, así es algo puntual, puede ser que se fijan menos. (1 Bach femenino) 
Según Raich, Mora y Soler (1994), se ha llegado a considerar que la presencia de la insatisfacción corporal especialmente en mujeres en la actualidad es una condición común, en el sentido de que la cultura actual demanda a las mujeres una creciente preocupación sobre la representación de la belleza femenina en ideales limitados a la apariencia física. También para las autoras feministas Etcoff, Orbach y Scout (2004), la mayoría de las mujeres ven la belleza y los atractivos físicos como altamente demandados y de reconocida importancia. De esta forma, sienten la presión social de ser físicamente más atractivas que la generación de sus madres, ya que los distintos ámbitos sociales están constantemente enviando mensajes en el sentido de mejorar cada vez más su apariencia.

De forma general, los participantes reconocen la influencia de los medios de comunicación, registrando que estos fortalecen un discurso en donde la apariencia física es lo más importante. Así, se construyen modelos de belleza, los cuales ya sea por omisión o por referencia explícita, también establecen lo que es feo. Además, apuntan que no sólo existe una divulgación de estos modelos, sino que también existe una tendencia hacia su imposición.

Esta imposición es sentida principalmente por las chicas en lo que se refiere a las tallas de las ropas.

Esto ya ha pasado por la tele que cada vez hace la ropa más pequeña y esto es malo, hasta para las modelos. ( $3^{\circ}$ ESO femenino)

A mí lo de las modelos no me parece tanto porque las modelos ya se saben, tienen que ser esto... modelos (risas). Pero lo malo es que vas a alguna tienda y no hay talla, todo es para tías minúsculas que no tienen pecho ni nada. (4 ESO femenino)

A mí, no me gusta ni me disgusta mi cuerpo, pero que quieres que te diga, si voy a la tienda y no puedo coger la 36 , pues hombre, no me sienta nada bien. ( $4^{\circ} \mathrm{ESO}$ femenino)

Ladrero (2005) afirma que desde principios de los años 90 las tallas (principalmente las destinadas a la población femenina) han sufrido importantes cambios, igualmente sujetos a condicionantes económicos, políticos, sociales y publicitarios. Si anteriormente la talla 44 era comercializada con normalidad en las tiendas de moda, ahora es difícil encontrar en muchas tiendas la talla 42. Por otro lado, destaca que antes la 38 era la talla mínima y si se llevaba menor talla había que ir a tiendas especializadas. Sin embargo, hoy en cualquier tienda de moda juvenil podemos encontrar las tallas 36, 34 e incluso 32, aunque muchas veces tales tallas no correspondan a medidas reales.

En 2008, el Ministerio de Sanidad y Consumo publicó el estudio antropométrico de la población femenina, cuyo objetivo era definir tres 
formas prevalecientes de silueta femenina, con el propósito de unificar las tallas de ropa en España. El estudio revela que el $80 \%$ de las mujeres participantes tiene un Índice de Masa Corporal considerado convencional y adecuado según parámetros estrictamente relativos a la salud. Sin embargo, el $40 \%$ de las mismas afirman tener problemas siempre o algunas veces para encontrar ropa de su talla, y el principal motivo, en todas las edades estudiadas, es que la ropa es pequeña. No obstante, en la actualidad, el proceso de unificación de tallas no se ha llevado a cabo por las firmas que suscribieron el acuerdo. El Ministerio de Sanidad y Consumo había asegurado que las tiendas tenían un plazo de cinco años para adaptarse a esta medida. De momento, ya han pasado algunos años y lo único que se ha avanzado es en el cambio de talla de los maniquíes, otro de los puntos con los que las empresas se comprometieron. Ahora usan una talla 38 en la mayoría de los establecimientos (FUNDACIÓN IMAGEN Y AUTOESTIMA, 2010).

\section{Conclusiones}

El concepto de imagen del cuerpo se ha recreado y modificado a lo largo de la historia humana y de acuerdo a determinadas culturas, pero actualmente existe un culto a la delgadez que, aunque no haya alcanzado a todas las sociedades, va en aumento, principalmente si tenemos en cuenta que a esta exigencia también se ha incorporado el proceso de globalización, que representa a las empresas transnacionales dedicadas a la venta de infinidades de productos destinados a lograr el ideal corporal.

Respecto a las diferencias de género, los participantes de los grupos de discusión son unánimes en indicar la existencia de mayor preocupación e insatisfacción con la imagen corporal en las chicas, que manifiestan sentimientos poco positivos hacia su propio cuerpo en comparación con los chicos. La insatisfacción corporal en las chicas se manifiesta en el deseo de perder peso y en los chicos se manifiesta en el deseo de ganar peso y musculatura.

Los modelos corporales que tienen como referencia derivan principalmente de los medios de comunicación, lo que se traduce en una complexión atlética para el hombre y mayor delgadez para la mujer. Los modelos corporales más destacados se refieren a cantantes, actrices, y modelos transmitidos por la publicidad y por la moda, aunque otros/as señalan la importancia de modelos corporales más cercanos y más reales, como parientes o amigos.

Los participantes de los grupos de discusión resaltan la importancia de los modelos socioculturales propagados por los medios de comunicación, 
así como de la influencia constante de estos medios, principalmente hacia la mujer. Señalan que el cuerpo delgado se asocia a una diversidad de significados que influyen en la construcción de la imagen corporal en la actualidad: la asociación entre delgadez y belleza, y entre belleza y éxito personal y profesional, así como la evidencia social de que las personas delgadas son mejor aceptadas en todos los ámbitos sociales, lo que implica también en la estigmatización de lo contrario.

Además, se manifiestan de forma contundente respecto a la inexistencia de la diversidad de tallas que se adapten a diferentes cuerpos, afirmando que las tallas son cada vez más pequeñas, y que la talla cambia según el modelo de ropa que se prueban. De esa forma, cuestionan la obligación de adaptarse a las tallas existentes y argumentan respecto al impacto psicológico negativo al no encontrar su talla.

En definitiva hemos podido comprobar la tremenda influencia que tienen los medios de comunicación respecto a los estereotipos de belleza corporal y de género, que son ampliadas por las presiones sociales del contexto en que los adolescentes se socializan y construyen sus imágenes de cuerpo. Es decir, en la cultura occidental contemporánea, las películas, la televisión, las revistas, la moda etc., envían el mensaje de que la belleza, el éxito, la felicidad personal, la juventud y la perfección están asociados a un prototipo corporal delgado. En este sentido, la imagen del cuerpo es dictada por una moda recogida del mundo externo y que gradualmente interiorizamos en nuestro imaginario individual y social como parte de lo que se espera de nosotros, lo que continúa afectando sobre todo a las mujeres.

IMAGEM CORPORAL E PERCEPÇÃO DA INFLUÊNCIA DA MÍDIA: DIFERENÇAS DE GÊNERO EM UMA AMOSTRA DE ADOLESCENTES

Resumo: O objetivo do estudo foi investigar as diferenças de gênero em uma amostra de adolescentes em relação à imagem corporal e à percepção da influência dos meios de comunicação. Foi realizado um estudo qualitativo na cidade de Toledo (Espanha), elegendo o grupo de discussão como técnica de coleta de dados. Foram constatadas diferenças nos discursos referentes ao corpo de acordo com o gênero e a presença de maior preocupação e insatisfação com a imagem corporal nas adolescentes, além da existência de diversidade de mediadores na construção da imagem corporal na atualidade: a norma da magreza, a inexistência de diversidade de tamanho de roupas que se adaptem a diferentes corpos, e a evidência social de que as pessoas magras são mais bem aceitas em todos os âmbitos sociais.

PALAVRAS-ChaVE: Imagem corporal. Gênero. Meios de comunicação de massa. 
BODY IMAGE AND PERCEIVING THE INFLUENCE OF THE MEDIA: GENDER DIFFERENCES IN A SAMPLE OF ADOLESCENTS

ABSTRACT: The aim of this study was to investigate gender differences in a sample of adolescents in relation to body image and perception of media influence. A qualitative study was conducted in Toledo (Spain), using the discussion group as a technique for data collection. Differences were found in the discourses with regard to the body according to gender and a greater concern and dissatisfaction with body image in female adolescents. A diversity of mediators in constructing the body image in today's world was also found. These include slimness being the norm, the lack of diversity of clothing sizes that can adapt to different bodies, and social evidence that slim people are better accepted in all social spheres.

KEY WORDS: Body image. Gender. Mass media.

\section{REFERÊNCIAS}

ALONSO, L. E. La mirada cualitativa en sociología. Madrid: Fundamentos, 1998.

BAILE AYENSA, J. I. B.; GUILLÉN, F.; GARRIDO, E. Insatisfacción corporal en adolescentes medida con el Body Shape Questionnaire (BSQ): efecto del anonimato, el sexo y la edad. International Journal of Clinical and Health Psychology, v. 2, n. 3, p. 439-450, feb./ mar. 2002.

CALADO, M.; LAMEIRAS, M.; SEPULVEDA, A. R.; RODRIGUEZ, Y.; CARRERA, M. V. The Association Between Exposure to mass media and body dissatisfaction among spanish adolescents. Women's Health Issues, v. 21, n.5, p. 390-399, 2011.

CASH, T. F.; PRUZINSKI, T. Integrative themes in body-image development, deviance and change. In: CASH, T. F.; PRUZINSKI, T. (Eds.). Body Images: development, deviance and change. New Yord: Guilford Press, 1990. p. 337-349.

ETCOFF, N.; ORBACH, S.; SCOTT, J. La verdad acerca de la belleza. Resultados del Estudio Global de Dove sobre las mujeres, la belleza y el bienestar, 2004. Disponível em: <http: www.porlabellezareal.com.ar/overview.asp?section=news $>$. Acesso em: 20 nov. 2010.

FUNDACIÓN IMAGEN Y AUTOESTIMA (2010). Análisis sobre el grado de unificación de tallas de ropa en España y el impacto en la salud de las personas. Barcelona: Fundación Imagen y Autoestima. Disponível en: <www.f-ima.org>. Acesso em: 2 ago. 2010.

GIDDENS, A. Modernidad e identidad del yo: el yo y la sociedad en la época contemporánea. Barcelona: Península, 1997.

GUTIÉRREZ, J. B. Dinámica del grupo de discusión. Madrid: CIS, 2008.

HARGREAVES, D. A.;TIGGEMANN, M. Female thin ideal media images and boys'attitudes toward girls. Sex Roles, v. 49, n. 9, p. 539-544, 2003.

HERNÁNDEZ, R.; FERNÁNDEZ, C.; BAPTISTA, P. Metodología de la investigación. México: Mc Graw Hill, 2006. 
IBÁÑEZ, J. Cómo se realiza una investigación mediante grupos de discusión. In: FERRANDO, G.; IBÁÑEZ, J.; ALVIRA, F. (Eds.). El análisis de la realidad social. Madrid: Alianza, 1994. p. 283-297.

LADRERO, E.U. En torno al vestido y al cuerpo: moda y aceptación social - años noventa y años dos mil. Zainak Cuadernos de Antropología-Etnografía, v. 27, p. 269-278, 2005.

LE BRETON, D. Antropología del cuerpo y modernidad. Buenos Aires: Nueva Visión, 2002.

LIPOVETSKY, G. La era del vacío: ensayos sobre el individualismo contemporáneo. Barcelona: Anagrama, 1986.

MINA-FREIRE, A. G. La construcción de una identidad personal en una cultura de género. Crítica, v. 55, n. 926, p. 45-48, 2005.

MURILLO, S. Relaciones de poder entre hombres y mujeres: los efectos del aprendizaje del rol en los conflictos y en la violéncia de género. Madrid: Federación de Mujeres Progresistas, 2000.

PARRA MARTÍNEZ, J. Análisis del sexismo en los libros de texto de Educación Física. Tesis Doctoral (Doctorado en Educación) - Universidad Nacional de Educación a Distancia, Madrid, ES, 2002.

PÉREZ GAULI, J. C. El cuerpo en venta. Madrid: Cátedra, 2000.

RAICH, R. M.; MORA, M.; SOLER, A. Revisión de la evaluación y tratamiento del trastorno de la imagen corporal y su adaptación en una muestra de estudiantes. Psicologemas, v. 8, n. 15, p. 81-99, 1994.

SAN MIGUEL, L. T. Mujer y filosofía. In: GONZÁLES, A.; LOMAS, L. (Eds.). Mujeryeducación: educar para la igualdad, educar desde la diferencia. Barcelona: Graó, 2002. p. 35-45.

SCHILDER, P. A imagem do corpo: as energias construtivas da psique. São Paulo: Martins Fontes, 1999.

SHERIFF, C. W. Needed concepts in the study of gender identity. Psychology of Women Quartery, v. 6, p. 375-398, 1982.

TAVARES, M. C. G. C. F. Imagem corporal: conceito e desenvolvimento. Barueri, SP: Manole, 2003.

WOLF, N. El mito de la belleza. Barcelona: Emecé, 1991.

WYKES, M.; GUNTER, B. The media \& body image: if looks could kill. London: Sage Pub, 2005.

Viviane Oliveira Gonçalves é doutora em Ciências do Esporte pela Universidad de Castilla-La Mancha, professora do Curso de Educação Física UFG/Campus Jataí.

E-mail: nanifer@hotmail.com 
Juan Parra Martínez é doutor em Ciências da Educação, professor da Faculdade de Ciências do Esporte, Universidad de Castilla-La Mancha (Espanha).

E-mail: JuanParra@uclm.es 